\title{
What Medication-Related Factors Are Important For Polypharmacy And Deprescribing Assessment By Physicians And Pharmacists Using A Novel Website To Rate Clinical Cases?
}

Nashwa Masnoon ( $\sim$ nashwa.masnoon@mymail.unisa.edu.au)

University of South Australia, Royal Adelaide Hospital https://orcid.org/0000-0002-7123-9583

Sepehr Shakib

Royal Adelaide Hospital

Lisa Kalisch Ellett

University of South Australia

Gillian E Caughey

South Australian Health and Medical Research Institute, University of South Australia, University of Adelaide, Royal Adelaide Hospital

\section{Research Article}

Keywords: polypharmacy, medication management, clinical pharmacy, medication harm, deprescribing, older adults, case-based assessment, risk assessment, clinician assessment of polypharmacy, physician assessment of polypharmacy, pharmacist assessment of polypharmacy

Posted Date: July 12th, 2021

DOI: https://doi.org/10.21203/rs.3.rs-395434/v1

License: (c) (i) This work is licensed under a Creative Commons Attribution 4.0 International License. Read Full License 


\section{Abstract}

Background: Little is known about how clinicians assess polypharmacy in real-world practice.

Objectives: To identify which medication-related factors influence clinicians' ratings of polypharmacy, harm from medicines and deprescribing of medicines when presented with a patient's list of medications and comorbidities.

Setting: A novel website called What Is Polypharmacy Exactly (WIPE) was created with 50 de-identified real-world cases with varying numbers of comorbidities and medicines.

Methods: Participants, consisting of physicians and pharmacists were asked to rate each case from zero (lowest) to 10 (highest) on three questions: i) degree of polypharmacy, ii) potential for medication-related harm and iii) potential to deprescribe medicines. Medication-related factors including medicines count, high-risk medicines, inappropriate duplication, drug-drug and drug-disease interactions were assessed. Multiple linear regression was used to determine which medication-related factors influenced median ratings for the three questions.

Main outcome measures: The primary outcome measure was the median rating for the degree of polypharmacy for each case. Secondary outcome measures were the median rating for the potential for harm from medicines for each case and median rating for the potential to deprescribe medicines for each case.

Results: Ninety-two clinicians were included in the study, comprising of $76.1 \%$ pharmacists $(n=70)$ and $23.9 \%$ physicians ( $n=22)$. The comorbidity count ( $P=0.001)$, medicines count $(P<0.001)$, inappropriate medication duplication $(P=0.017)$, high-risk medicines $(P=0.049)$, use of NSAIDs $(P=0.032)$ and antihypertensives $(P=0.040)$ were predictive of the perceived degree of polypharmacy. The comorbidity count $(P<0.001)$, medicines count $(P<0.001)$, inappropriate medication duplication $(P=0.016)$ and high-risk medicines $(P=0.036)$ were predictive of the recognised potential for medication-related harm. The medicines count $(P<0.001)$, inappropriate medication duplication $(P=0.017)$, benzodiazepine use $(P=0.003)$ and antipsychotic use $(P=0.039)$ were predictive of the recognised potential to deprescribe medicines.

Conclusion: Whilst polypharmacy has traditionally been defined using the medicines count, our findings suggest that clinicians also consider other factors such as high-risk medicines and inappropriate medicines duplication to identify patients at risk of adverse outcomes. Future polypharmacy assessment tools should additionally include these factors.

\section{Impact Of Findings On Practice}

- Whilst polypharmacy research has traditionally focused on the number of medicines, our findings demonstrate that when clinicians assess polypharmacy, potential from harm from medicines and potential to deprescribe medicines, they additionally consider other medication-related factors such as the use of high-risk medicines and inappropriate medication duplication.

- Medical practitioners, pharmacists and other healthcare professionals in practice who treat multimorbid older adults with polypharmacy and develop future polypharmacy tools and guidelines, should consider factors such as high risk medicines use and presence of inappropriate medication duplication, when assessing polypharmacy.

\section{Introduction}

The world's population is ageing and the proportion of the population aged 60 years and over is estimated to nearly double from $12 \%$ in 2015 to $22 \%$ in 2050 [1]. The proportion of people living with multiple chronic conditions, termed multimorbidity is subsequently increasing $[2,3]$. As a result, the use of multiple medicines or polypharmacy is common, particularly in the older population. Older patients are at high risk of adverse outcomes from polypharmacy due to age-related changes in pharmacokinetics and pharmacodynamics [4, 5]. Polypharmacy has been associated with a range of adverse outcomes including adverse drug reactions, unplanned hospitalisations, frailty, falls, decline in cognition, renal impairment and mortality [6-8]. Harm from polypharmacy is a multifaceted problem, with issues such as poor communication and handover between different specialties and professions, especially during transitions of care compounding the problem [9].

A systematic review of the definitions of polypharmacy revealed a lack of consensus regarding how polypharmacy is defined, making it challenging for healthcare professionals to assess the appropriateness of polypharmacy in the clinical setting [10]. The most commonly reported definition of polypharmacy was the use of five or more medicines [10]. This definition does not provide a meaningful evaluation of the clinical appropriateness of polypharmacy.

In the face of multiple definitions of polypharmacy which are not clinically meaningful for polypharmacy rationalisation, it remains unclear what medicationrelated factors clinicians consider during polypharmacy assessment. Various drug classes such as opioids, benzodiazepines, anticholinergic drugs, antipsychotics, antidepressants, antihypertensives, nonsteroidal anti-inflammatory drugs (NSAIDs) and antiepileptics have been associated with adverse outcomes such as falls, adverse drug reactions and hospitalisations [11-15]. However, the studies identifying these associations have been conducted across a range of age groups, including younger adults, limiting their applicability to the older population. It is unclear as to which of these drug classes resonate with clinicians as having high likelihood to cause harm in the older population. Minimising the potential for adverse outcomes from medicines associated with harm involves exploring the possibility of deprescribing [16]. It is unclear however which medicines clinicians actually prioritise for deprescribing. An understanding of the key medication-related factors that clinicians use to assess polypharmacy, which medicines they identify as having high risk of harm and which medicines they prioritise for deprescribing would be useful to inform future polypharmacy assessment tools and guidelines. In Australia 250,000 
hospital admissions annually are a result of medication-related problems, costing $\$ 1.4$ billion annually [17]. There are 400,000 additional presentations to emergency departments which are likely to be due to medication-related problems [17].

Medications are managed by a multidisciplinary team in Australia [18]. Whilst physicians have the authority to make changes to medications, such as adding or ceasing medications and increasing or decreasing doses of medications, pharmacists also have an active role in polypharmacy assessment and rationalisation in different settings such as hospital, community and nursing homes. Pharmacists undertake different tasks from undertaking an accurate medication history and clinical review to recommending medication changes to physicians, supplying different medications and educating patients on how to best manage different medications, including monitoring for potential side effects. As a result, geriatric medication management is undertaken by both physicians and pharmacists as a multidisciplinary team in Australia. As part of recommending medication changes to physicians after conducting a medication review, pharmacists suggest the prescribing or deprescribing of different medications, as per the patient's presenting complaint, patient's goals and preferences, medical conditions, allergies and intolerances, blood test results and other clinical information. In community pharmacies, pharmacists additionally recommend over the counter medications for customers presenting with minor ailments such as coughs and colds, skin disorders and headache.

Previous studies have identified factors important for polypharmacy assessment through survey of the literature and surveying expert clinicians in geriatric medicine [21,33]. These factors include indications of different medicines, use of high risk medicines which have high potential for harm such as anticoagulants and insulin, drug-drug and drug-disease interactions [21,33]. These studies however did not assess how clinicians assess polypharmacy, using de-identified patient cases, to simulate clinical practice. The aim of this study was to identify which medication-related factors influence assessment of polypharmacy, potential for harm from medicines and potential to deprescribe medicines by clinicians when presented with a patient's list of medications and chronic conditions, using de-identified cases from clinical practice.

\section{Methods}

\subsection{Development of a platform for polypharmacy assessment}

A novel, web-based platform called What Is Polypharmacy Exactly (WIPE) was developed at wipe.logicsquad.net/signup. The research team consisting of the study authors, built WIPE with help from the software development team called Logic Squad. WIPE was developed to assess how clinicians view polypharmacy, as part of the ongoing research undertaken by the Department of Clinical Pharmacology at the Royal Adelaide Hospital into which medicationrelated factors are important for polypharmacy assessment, from the perspective of clinicians (WIPE study) and using patient outcome data.

Fifty de-identified cases based on real patient scenarios from an outpatient multimorbidity clinic [19] at the Royal Adelaide Hospital were uploaded on to WIPE. Cases were selected by two investigators (a clinical pharmacologist and clinical pharmacist) to ensure an approximately even distribution across different tertiles of the total number of medicines (including regular, as needed and complementary), namely minimal (one to six medicines), medium (seven to 10 medicines) and high (11 or more medicines) as shown in Figure 1. Cases were also selected to ensure a range of medicines were covered including cardiovascular, neurological and psychotropic drugs. WIPE allows users to choose how many cases they rate. Therefore, not all users rated all cases, meaning some cases had a higher number of ratings and others had a lower number of ratings. The cases with the least number of ratings at the time of the user logging in present first to ensure all cases receive user ratings.

Figure 2 shows a snapshot of a WIPE case, patient demographics were presented followed by a list of medications and chronic conditions. The patient demographics for all cases were kept constant: "A 67 year old person living independently in the community", to ensure the identification of medication-related factors affecting polypharmacy assessment, independent of a patient's age and setting.

Users were asked to rate each case from 0 (lowest) to 10 (highest) for the following three questions:

Question 1: the degree of polypharmacy

Question 2: the potential for harm from medications

Question 3: the potential to deprescribe medications

\subsection{Participant recruitment and data collection}

Participant recruitment for WIPE involved emails to clinicians working in the area of geriatric medicine as well as promoting WIPE via newsletters, podcasts and conference presentations for different pharmacy associations. A Twitter account was also opened to promote WIPE using the username of WIPEProject. No limit was placed on the area of expertise of WIPE users, to ensure a wide range of participants were included. Medical and pharmacy students who signed up on WIPE were excluded from the study, as we wanted users who had at least completed a bachelor's degree in medicine or pharmacy to rate cases on WIPE. Data collected on WIPE from July 2017 to July 2018 was analysed for this study. Given both pharmacists and physicians play an active role in medication management, their results were pooled together for this study.

\subsection{Outcome variables}

The primary outcome measure was the median rating for the degree of polypharmacy for each case. Secondary outcome measures were the median rating for the potential for harm from medicines for each case and median rating for the potential to deprescribe medicines for each case.

3.4 Determination of medication-related and diseaserelated factors 
For each case we determined a number of medication-related factors and performed univariate analysis to assess their relationship to clinician assessment of the three outcome variables. Medication-related factors were shortlisted from a systematic review of polypharmacy definitions [10], polypharmacy assessment tools and criteria [20] and combined with findings from a survey of clinical experts regarding medication-related factors which are important for polypharmacy rationalisation [21].

Medication-related factors included the total number of medicines used by a patient which included over the counter, as needed and complementary and alternative medicines. The number of medicines within specific medicine classes which are associated with harm such as anticoagulants, sedatives or anticholinergics were also included as medication-related factors. High-risk medicines were also included as a group. Medicines which are less commonly associated with harm, namely paracetamol, vitamin D and multivitamins were also included as negative controls. These medications are usually considered to have relatively lower potential for harm and therefore, if the results indicate that these medications are not commonly associated with harm by clinicians, this would provide validation of our findings [21]. The number of medicines within each individual medication class was identified using the Australian Medicines Handbook (AMH) [22]. The number of high-risk medicines were identified using APINCHEN, which is an acronym developed by the South Australian Health Department, used to identify high-risk medicines which have the potential to cause significant harm if misused or administered incorrectly [20]. Sedatives were identified using the 'Narcotics and other sedatives' section of the APINCHEN [20]. Anticholinergics were identified using the 'Drugs with anticholinergic effects' table from the $\mathrm{AMH}$ [22].

Other medication-related factors included the number of identified drug-drug and drug-disease interactions as well as inappropriate drug class duplication. Drug-drug interactions were identified via the Australian Medicines Handbook [22]. Inappropriate drug class duplication was also defined via the AMH using the 'Drug treatment' or 'Drug choice' section for each disease [22]. An example of an inappropriate drug class duplication is the use of two calcium channel blockers concurrently. In contrast, a potentially appropriate duplication would be the concurrent use of a regular slow release opioid with an as needed immediate release opioid for breakthrough pain. Drug-disease interactions were identified using the list of drug-disease or drug-syndrome interactions from Beers Criteria 2015 (latest version available at the time of the study) [24] and a list of treatment conflicts shortlisted after reviewing Australian clinical guidelines on diabetes, heart failure and chronic obstructive pulmonary disease [25-27]. The complete list of medication-related variables and how they were identified is summarised in Appendix A. Medication-related factors which relate to medications in combination with each other or medications in combination with diseases, namely drug-drug and drug-related interactions and inappropriate drug class duplication were categorised under 'Medication-combination variables' in each of the tables. Individual drug classes such as benzodiazepines or opioids and groups of drug classes such as high risk medicines are categorised under 'Drug classes individually or grouped' in each of the tables.

The disease-related factor included in this study was the comorbidity count. This was determined using the list of chronic conditions for each patient. For example, for a patient with rheumatoid arthritis, depression and hypertension, the comorbidity count would be three.

\subsection{Factors influencing ratings for polypharmacy assessment, potential for harm from medicines and potential to deprescribe medicines}

Disease-related (comorbidity count) and medication-related factors which were significant with a $p$-value $\leq 0.20$ after univariate analysis were included in multiple linear regression against the three outcomes, to identify which factors influence ratings for the degree of polypharmacy, potential for harm from medicines and potential to deprescribe medicines. Medication-related factors which include individual drug classes were not included in the same model as those individual medication classes. For example, high risk medicines include the individual drug class of anticoagulants. As a result, high risk medicines and anticoagulants were not included the same model.

\subsection{Current definitions of polypharmacy as comparators}

Comparator models were included to assess how the addition of different medication-related factors to the standard definition of polypharmacy impacted the goodness of fit of the final models. We included as comparator models the total number of medicines (comparator 1) and the use of five or more medicines (comparator 2) as both of these have been used to traditionally define polypharmacy in the literature [10]. Another comparator model included the total number of medicines and the comorbidity count, if the comorbidity count is significant after multivariate analysis for each of the three questions (comparator 3).

\subsection{Statistical analysis}

Data are presented as medians with interquartile ranges (IQRs) and percentages. Medication-related variables individually associated with the three outcome measures were assessed using the Pearson's correlation for normally distributed data and Spearman's correlation for non-normally distributed data. Tolerance and Variance Inflation Factor (VIF) were used to test for multicollinearity between shortlisted independent variables. A tolerance of less than 0.1 and VIF value exceeding 10 were considered as the presence of multicollinearity. Factors which had a $p$-value $\leq 0.2$ after correlation analysis were shortlisted for inclusion in multiple linear regression.

Multiple linear regression was conducted using the enter method with a significance level of $p$-value $<0.05$ for inclusion in the final model. $\mathrm{R}^{2}$ values were used to assess goodness of fit of different models.

All analyses were undertaken using SPSS version 25 (IBM Corp. Released 2017. IBM SPSS Statistics for Windows, Version 25.0. Armonk, NY: IBM Corp.).

\subsection{Ethics approval}

This study was approved by the Human Research Ethics Committees at the Royal Adelaide Hospital (R20170705) and the University of South Australia (200260). 


\section{Results}

\subsection{User and case characteristics}

A total of 92 users rated WIPE cases comprising of $76.1 \%$ pharmacists $(n=70)$ and $23.9 \%$ physicians $(n=22)$. The highest number of ratings received for a case was 36 and lowest number of ratings received for a case was 18, with the median number of case ratings being 26.

As shown in Table 1, for the 50 cases included on WIPE, the median number of comorbidities was 5.5 (IQR 4.0 - 7.3 ) and medications was 8.0 (IQR 5.8 12.5). With regard to medication-combination variables, drug-drug interactions were most common, with $68 \%$ ( $n=34$ ) of cases having drug-drug interactions. With regard to drug classes grouped, $60 \%(n=30)$ of cases contained high risk medications. In terms of individual drug classes, antihypertensives were most common, with $66 \%(n=33)$ of cases having antihypertensives.

Table 1. Disease and medication-related characteristics of patient cases $(n=50)$

\begin{tabular}{|c|c|}
\hline Variable & $\begin{array}{l}\text { Median (IQR) for each variable or } \\
\text { Number of cases with each variable (\% of cases with each variable) }\end{array}$ \\
\hline \multicolumn{2}{|l|}{ Disease-related variable } \\
\hline Comorbidity count & $5.5(4.0-7.3)$ \\
\hline \multicolumn{2}{|l|}{ Medication-related variables } \\
\hline \multicolumn{2}{|l|}{ Number-related variable } \\
\hline Total number of medicines & $8.0(5.8-12.5)$ \\
\hline \multicolumn{2}{|l|}{ Medication-combination variables } \\
\hline Drug-drug interaction & $34(68.0)$ \\
\hline Drug-disease interaction & $12(24.0)$ \\
\hline Inappropriate drug class duplication & $6(12.0)$ \\
\hline \multicolumn{2}{|l|}{ Drug classes individually or grouped } \\
\hline High-risk Medicine & $30(60.0)$ \\
\hline Benzodiazepine & $7(14.0)$ \\
\hline Opioid & $9(18.0)$ \\
\hline Anticholinergic & $15(30.0)$ \\
\hline Sedative or anticholinergic & $26(52.0)$ \\
\hline Digoxin & $2(4.0)$ \\
\hline Antiarrhythmic & $6(12.0)$ \\
\hline Anticoagulant & $13(26.0)$ \\
\hline Antiplatelet & $19(38.0)$ \\
\hline Antihypertensive & $33(66.0)$ \\
\hline Diuretic & $19(38.0)$ \\
\hline Insulin & $5(10.0)$ \\
\hline Oral hypoglycaemic & $12(24.0)$ \\
\hline Systemic corticosteroid & $3(6.0)$ \\
\hline Antipsychotic & $6(12.0)$ \\
\hline Antidepressant & $15(30.0)$ \\
\hline Antiepileptic & $11(22.0)$ \\
\hline Antiparkinson & $3(6.0)$ \\
\hline NSAID & $3(6.0)$ \\
\hline Paracetamol & $24(48.0)$ \\
\hline Vitamin D & $13(26.0)$ \\
\hline Multivitamin & $3(6.0)$ \\
\hline
\end{tabular}


Examples of variables which were significantly associated with the degree of polypharmacy, potential for harm from medicines and potential to deprescribe medicines during univariate analysis at a significance level of $P \leq 0.20$, as rated by clinicians are the comorbidity count, total number of medicines, drug-drug and drug-disease interactions, inappropriate drug class duplication, high risk medicines, opioids, benzodiazepines, antipsychotics and NSAIDs. The full list of variables which were individually associated with ratings for each of the three outcomes is shown in Table 2.

Table 2. Correlations of individual variables and median ratings for each of the three questions for each case 


\begin{tabular}{|c|c|c|c|c|}
\hline Variable & $\begin{array}{l}R \text { and } p- \\
\text { values }\end{array}$ & $\begin{array}{l}\text { Polypharmacy } \\
\text { score }\end{array}$ & $\begin{array}{l}\text { Potential for harm from } \\
\text { medications }\end{array}$ & $\begin{array}{l}\text { Potential to deprescribe } \\
\text { medications }\end{array}$ \\
\hline \multicolumn{5}{|l|}{ Disease-related variable } \\
\hline \multirow[t]{2}{*}{ Comorbidity count } & $\mathrm{R}$ & 0.49 & 0.40 & 0.23 \\
\hline & P-value & 0.001 & 0.004 & 0.11 \\
\hline \multicolumn{5}{|l|}{ Medication-related variables } \\
\hline \multicolumn{5}{|l|}{ Number-related variable } \\
\hline \multirow[t]{2}{*}{ Total number of medicines } & $\mathrm{R}$ & 0.70 & 0.63 & 0.55 \\
\hline & P-value & $<0.001$ & $<0.001$ & $<0.001$ \\
\hline \multicolumn{5}{|c|}{ Medication-combination variables } \\
\hline \multirow[t]{2}{*}{ Drug-drug interaction } & $\mathrm{R}$ & 0.27 & 0.22 & 0.22 \\
\hline & P-value & 0.06 & 0.13 & 0.13 \\
\hline \multirow[t]{2}{*}{ Drug-disease interaction } & $\mathrm{R}$ & 0.29 & 0.24 & 0.20 \\
\hline & P-value & 0.04 & 0.10 & 0.16 \\
\hline \multirow{2}{*}{$\begin{array}{l}\text { Inappropriate drug class } \\
\text { duplication }\end{array}$} & $\mathrm{R}$ & 0.32 & 0.30 & 0.39 \\
\hline & P-value & 0.03 & 0.03 & 0.005 \\
\hline \multicolumn{5}{|c|}{ Drug classes individually or grouped } \\
\hline \multirow[t]{2}{*}{ High Risk Medicine } & $\mathrm{R}$ & 0.56 & 0.61 & 0.43 \\
\hline & P-value & $<0.001$ & $<0.001$ & 0.002 \\
\hline \multirow[t]{2}{*}{ Benzodiazepine } & $\mathrm{R}$ & 0.20 & 0.25 & 0.29 \\
\hline & P-value & 0.16 & 0.08 & 0.04 \\
\hline \multirow[t]{2}{*}{ Opioid } & $\mathrm{R}$ & 0.42 & 0.41 & 0.23 \\
\hline & P-value & 0.002 & 0.003 & 0.11 \\
\hline \multirow[t]{2}{*}{ Anticholinergic } & $\mathrm{R}$ & 0.20 & 0.21 & 0.26 \\
\hline & P-value & 0.16 & 0.14 & 0.07 \\
\hline \multirow[t]{2}{*}{ Sedative or anticholinergic } & $\mathrm{R}$ & 0.44 & 0.51 & 0.47 \\
\hline & P-value & 0.001 & $<0.001$ & 0.001 \\
\hline \multirow[t]{2}{*}{ Digoxin } & $\mathrm{R}$ & 0.13 & 0.18 & 0.09 \\
\hline & P-value & 0.38 & 0.22 & 0.56 \\
\hline \multirow[t]{2}{*}{ Antiarrhythmic } & $\mathrm{R}$ & 0.10 & 0.14 & 0.01 \\
\hline & P-value & 0.50 & 0.33 & 0.94 \\
\hline \multirow[t]{2}{*}{ Anticoagulant } & $\mathrm{R}$ & 0.13 & 0.25 & 0.11 \\
\hline & P-value & 0.37 & 0.08 & 0.44 \\
\hline \multirow[t]{2}{*}{ Antiplatelet } & $\mathrm{R}$ & 0.11 & -0.07 & 0.08 \\
\hline & P-value & 0.43 & 0.65 & 0.59 \\
\hline \multirow[t]{2}{*}{ Antihypertensive } & $\mathrm{R}$ & 0.24 & 0.15 & 0.15 \\
\hline & P-value & 0.09 & 0.29 & 0.29 \\
\hline \multirow[t]{2}{*}{ Diuretic } & $\mathrm{R}$ & 0.26 & 0.19 & 0.04 \\
\hline & P-value & 0.07 & 0.19 & 0.77 \\
\hline \multirow[t]{2}{*}{ Insulin } & $\mathrm{R}$ & 0.14 & -0.02 & 0.002 \\
\hline & P-value & 0.35 & 0.91 & 0.99 \\
\hline \multirow[t]{2}{*}{ Oral hypoglycaemic } & $\mathrm{R}$ & 0.24 & 0.16 & 0.12 \\
\hline & P-value & 0.097 & 0.261 & 0.422 \\
\hline Systemic corticosteroid & $\mathrm{R}$ & -0.08 & -0.009 & -0.04 \\
\hline
\end{tabular}

Page $7 / 13$ 


\begin{tabular}{|c|c|c|c|c|}
\hline Variable & $\begin{array}{l}R \text { and } p- \\
\text { values }\end{array}$ & $\begin{array}{l}\text { Polypharmacy } \\
\text { score }\end{array}$ & $\begin{array}{l}\text { Potential for harm from } \\
\text { medications }\end{array}$ & $\begin{array}{l}\text { Potential to deprescribe } \\
\text { medications }\end{array}$ \\
\hline & P-value & 0.60 & 0.95 & 0.79 \\
\hline \multirow[t]{2}{*}{ Antipsychotic } & $\mathrm{R}$ & 0.25 & 0.34 & 0.43 \\
\hline & P-value & 0.08 & 0.02 & 0.002 \\
\hline \multirow[t]{2}{*}{ Antidepressant } & $\mathrm{R}$ & 0.57 & 0.58 & 0.49 \\
\hline & P-value & $<0.001$ & $<0.001$ & $<0.001$ \\
\hline \multirow[t]{2}{*}{ Antiepileptic } & $\mathrm{R}$ & 0.28 & 0.23 & 0.27 \\
\hline & P-value & 0.05 & 0.10 & 0.06 \\
\hline \multirow[t]{2}{*}{ Antiparkinson } & $\mathrm{R}$ & 0.27 & 0.21 & 0.11 \\
\hline & P-value & 0.06 & 0.15 & 0.46 \\
\hline \multirow[t]{2}{*}{ NSAID } & $\mathrm{R}$ & 0.29 & 0.29 & 0.20 \\
\hline & P-value & 0.04 & 0.04 & 0.17 \\
\hline \multirow[t]{2}{*}{ Paracetamol } & $\mathrm{R}$ & 0.28 & 0.29 & 0.07 \\
\hline & P-value & 0.05 & 0.04 & 0.62 \\
\hline \multirow[t]{2}{*}{ Vitamin D } & $\mathrm{R}$ & 0.20 & 0.13 & 0.15 \\
\hline & P-value & 0.16 & 0.38 & 0.30 \\
\hline \multirow[t]{2}{*}{ Multivitamin } & $\mathrm{R}$ & 0.14 & 0.04 & -0.09 \\
\hline & P-value & 0.34 & 0.79 & 0.54 \\
\hline
\end{tabular}

4.3 Factors influencing ratings for the degree of polypharmacy, potential for harm from medicines and potential to deprescribe medicines

Multivariate analysis demonstrated that comorbidity count $(P=0.001)$, total number of medicines $(P<0.001)$, inappropriate drug class duplication $(P=$ 0.017), use of high-risk medicines $(P=0.049)$, NSAIDs $(P=0.032)$ and antihypertensives $(P=0.040)$ were predictive of the degree of polypharmacy as rated by the clinicians $\left(\mathrm{R}^{2}=0.94\right)$ as shown in Table 3 . The comorbidity count $(P<0.001)$, total number of medicines $(P<0.001)$, inappropriate drug class duplication $(P=0.016)$ and use of high-risk medicines $(P=0.036)$ were predictive of the potential for harm from medicines $\left(\mathrm{R}^{2}=0.93\right)$. The total number of medicines $(P<0.001)$, inappropriate drug class duplication $(P=0.017)$, benzodiazepines $(P=0.003)$ and antipsychotics $(P=0.039)$ were predictive of the potential to deprescribe medicines $\left(R^{2}=0.89\right)$.

Table 3. Factors predictive of median ratings for each of the three questions for each case (multiple linear regression) 


\begin{tabular}{|c|c|c|c|c|}
\hline Variable & $\mathrm{R}^{2}$ & Standardised beta (coefficient) & $\mathrm{t}$ & P-value \\
\hline \multicolumn{5}{|c|}{ Factors predictive of the degree of polypharmacy (question one) } \\
\hline Comorbidity count & \multirow[t]{6}{*}{0.94} & 0.30 & 3.68 & 0.001 \\
\hline Total number of medicines & & 0.46 & 5.09 & $<0.001$ \\
\hline Inappropriate drug class duplication & & 0.10 & 2.47 & 0.017 \\
\hline High-risk medicine & & 0.11 & 2.03 & 0.049 \\
\hline NSAID & & 0.09 & 2.21 & 0.032 \\
\hline Antihypertensive & & 0.11 & 2.11 & 0.040 \\
\hline \multicolumn{5}{|c|}{ Factors predictive of the potential for harm from medications (question two) } \\
\hline Comorbidity count & \multirow[t]{4}{*}{0.93} & 0.34 & 3.80 & $<0.001$ \\
\hline Total number of medicines & & 0.50 & 5.17 & $<0.001$ \\
\hline Inappropriate drug class duplication & & 0.11 & 2.50 & 0.016 \\
\hline High-risk medicines & & 0.13 & 2.16 & 0.036 \\
\hline \multicolumn{5}{|c|}{ Factors predictive of the potential to deprescribe medications (question three) } \\
\hline Total number of medicines & \multirow[t]{4}{*}{0.89} & 0.78 & 14.48 & $<0.001$ \\
\hline Inappropriate drug class duplication & & 0.13 & 2.47 & 0.017 \\
\hline Benzodiazepine $^{a}$ & & 0.16 & 3.15 & 0.003 \\
\hline Antipsychotic $^{a}$ & & 0.11 & 2.13 & 0.039 \\
\hline
\end{tabular}

aWhilst high-risk medicines as a group were predictive of the degree of polypharmacy (question one) and potential for harm from medicines (question two), individual drug classes, namely benzodiazepines and antipsychotics were predictive of the potential to deprescribe medicines (question three)

\subsection{Current definitions of polypharmacy as comparators}

The $\mathrm{R}^{2}$ value for comparator 1 (total number of medicines) was 0.90 , comparator 2 (use of five or more medicines) was 0.87 and comparator 3 (comorbidity count and total number of medicines) was 0.92 for the degree of polypharmacy, compared to 0.94 for our model. The $R^{2}$ value for comparator 1 was 0.89 , comparator 2 was 0.87 and comparator 3 was 0.91 for the potential for harm from medicines, compared to 0.93 for our model. The $R^{2}$ value for comparator 1 was 0.85 and comparator 2 was 0.84 for the potential to deprescribe medicines, compared to 0.89 for our model. Comparator 3 which includes the comorbidity count and total number of medicines was not applicable to this question as comorbidity count was not significant after multivariate analysis.

\section{Discussion}

This is the first study to undertake a thorough assessment of various medication-related factors against ratings by clinicians for polypharmacy, the potential for harm from medicines and potential to deprescribe medicines, using cases based on real-world patients. A systematic review of polypharmacy definitions revealed that whilst there is no consensus regarding the definition of polypharmacy, the most common definition is the use of five or more medicines [10]. Our findings provide guidance for the inclusion of other medication-related factors, including use of high risk medicines and inappropriate medication duplication when developing future polypharmacy tools and deprescribing guidelines. These results align with recent literature which has suggested to look beyond simply the number of medicines and incorporate the concept of the quality of prescribing by considering specific types of medicines and inappropriate medication duplication to assess polypharmacy appropriateness [10,21, 28-31]. Additionally, these findings provide guidance for identifying patients who may require a comprehensive review of their medicines such as a home medicines review (HMR) by a pharmacist [32]. A HMR is a service in Australia where a pharmacist visits a patient in their home and goes through all medicines being used to undertake a comprehensive review of the different medications being used. Previously, a patient was required to be on five or more medicines to be eligible for a HMR. The criteria were subsequently changed to patients who are at risk of "medication misadventure" in order to be eligible for a HMR. However it is still unclear what criteria constitutes "medication misadventure". Clinicians in our study nominated the use of high-risk medicines (which includes anticoagulants, opioids and benzodiazepines) as well as the comorbidity count, number of medicines and inappropriate medicines duplication as being important for the potential for harm from medicines. Therefore, this combination of comorbidities, number and types of medicines can provide more specific guidance for General Practitioners regarding which patients to refer for HMRs.

Inappropriate medication duplication, the use of benzodiazepines, antipsychotics and the total number of medicines were significantly associated with the potential to deprescribe. Inappropriate medication duplication, high risk medicines (which include benzodiazepines) and number of medicines were also predictive of the potential for harm from medicines. These results align with the risk minimisation approach of identifying medicines associated with harm and exploring the possibility of deprescribing them or switching to a safer alternative. Our findings further confirm this process from the perspective of a large number and range of clinicians. Previous studies of clinical experts in geriatric medicine have found strong consensus regarding considering medicationrelated factors such as the use of high-risk medicines including benzodiazepines and antipsychotics as well as inappropriate duplication of medicines in addition to the medicines count for polypharmacy rationalisation [21,30,33]. Our findings combined with previous literature provide guidance for pharmacists 
undertaking home medicines reviews in the community and nursing home settings and clinicians undertaking medication reviews in the hospital setting, to identify medicines commonly associated with harm and exploring the possibility of deprescribing them. Key targets include benzodiazepines and antipsychotics which have higher likelihood to cause adverse outcomes in the older population.

The $\mathrm{R}^{2}$ values for models incorporating factors predictive of the degree of polypharmacy, potential for harm from medicines and potential to deprescribe medicines were marginally higher than the different comparators included in this study. Therefore, these results align with recent literature regarding looking beyond just the medicines count as the models incorporating other medication-related factors better predicted the three outcomes, compared to comparator models, which were based on the number of medicines. Future tools and guidelines aimed at polypharmacy rationalisation should therefore consider a combination of comorbidities, number and types of medicines to identify patients at risk of adverse outcomes. Future research should also undertake a thorough assessment of which medication-related factors are actually associated with patient-related outcomes such as hospitalisation and mortality. These findings may also provide guidance for clinicians assessing and minimising harm from polypharmacy in actual clinical practice. Clinicians should not only consider the number of medications but also consider comorbidities, presence of inappropriate drug class duplication and the use of high risk medicines when identifying the potential harm from multiple medicines use.

Strengths of our study include the use of real-world de-identified cases from a multimorbidity clinic. Secondly, the inclusion of negative controls such as paracetamol and vitamin $\mathrm{D}$, which did not remain significant after multiple linear regression analysis further validates our findings. Thirdly, an approximately even distribution of the number of medicines across the different tertiles in the different cases ensures applicability to current practice. Limitations include the uneven distribution of comorbidities across the different tertiles, despite the approximately even distribution of the number of medicines. WIPE allows users to choose how many cases they rate. Therefore each case does not represent perspectives of all the different users involved. There is the possibility that respondents were getting accustomed to the WIPE website during the first few cases and getting fatigued when rating the last few cases, meaning there may be ratings which do not accurately reflect user perspectives. Another limitation of this study is the higher proportion of pharmacists compared to physicians. Whilst both professions are part of the multidisciplinary team managing patient's medication, physicians have prescribing and deprescribing rights and ultimately decide whether medications are started or deprescribed. Future research may help elucidate perspectives on polypharmacy, potential for harm and potential to deprescribe medications from the perspectives of physicians compared to pharmacists. Lastly, during the signing up process, WIPE users were asked to indicate their years of experience. This was however not a mandatory field. As a result, this information was not available for all users. Future research could explore perspectives of different clinicians according to clinician characteristics, such as years of experience and area of practice.

Whilst WIPE was initially designed to assess clinician perspectives on polypharmacy, potential for harm from medications and potential to deprescribe medications, WIPE has now become an educational resource. Undergraduate medical and pharmacy students rate WIPE cases with subsequent group discussion regarding ratings for the three outcomes and the reasons behind their ratings based on the medications and comorbidities. Being a web-based platform, WIPE has the potential to bring together perspectives of different clinicians on polypharmacy assessment across Australia and internationally.

\section{Conclusions}

Whilst polypharmacy has mainly been defined using the number of medicines, our findings suggest that other medication-related factors, such as the use of high-risk medicines and inappropriate medication duplication are important when clinicians assess polypharmacy, potential from harm from medicine regimens and potential to deprescribe. These factors provide guidance for risk stratifying older patients who may need further assessment of their medicines such as home medicines reviews. Our findings also provide guidance for which medicines to target during deprescribing interventions, such as benzodiazepines and antipsychotics, both of which are known to be associated with harm, particularly in the older population. Future studies should assess which medication-related factors are associated with patient-related outcomes such as hospitalisation and mortality.

\section{Declarations}

\section{Data Availability Statement}

Majority of data generated or analysed during this study are included in this published article. Any other data generated during and/or analysed during the current study are available from the corresponding author on reasonable request.

\section{Code availability}

Not applicable

\section{Conflicts of interest}

All authors (NM, SS, LK and GC) have no conflicts of interest to declare.

\section{Sources of funding}

NM was supported by an Australian Government Research Training Program whilst this study was undertaken.

\section{Ethics approval}

This study was approved by the Human Research Ethics Committees at the Royal Adelaide Hospital (R20170705) and the University of South Australia (200260). 


\section{Consent to participate}

Users were invited to sign up themselves if they consented to participate. Therefore, signing up on as a user on WIPE was accepted as providing consent to participate in the WIPE project.

\section{Consent for publication}

Prior to signing up on the WIPE website, users were advised that the data generated from the WIPE project would be used for scientific publication and therefore by signing up, they provided consent to participate and consent for publication.

Acknowledgements

NM was supported by the Australian Government Research Training Program when undertaking the research for this paper. NM undertook the data analysis with supervision from GC, SS and LK. NM drafted the initial manuscript with subsequent revisions for the other three authors. All authors consented to the final version of the manuscript.

\section{References}

1. World Health Organization. Ageing and health. 2018. http://www.who.int/news-room/fact-sheets/detail/ageing-and-health. Accessed 20-07-18 .

2. Fabbri E, Zoli M, Gonzalez-Freire M, Salive ME, Studenski SA, Ferrucci L. Aging and Multimorbidity: New Tasks, Priorities, and Frontiers for Integrated Gerontological and Clinical Research. J Am Med Dir Assoc . 2015;16(8):640-7.

3. World Health Organization. Global health and ageing. 2011. https://www.who.int/ageing/publications/global_health.pdf?ua=1

4. Corsonello A, Pedone C, Incalzi RA. Age-related pharmacokinetic and pharmacodynamic changes and related risk of adverse drug reactions. Curr Med Chem. 2010;17(6):571-84.

5. Pont LG, McLachlan AJ. Drug Metabolism in Older People-A Key Consideration in Achieving Optimal Outcomes With Medicines. The Journals of Gerontology: Series A. 2011;67A(2):175-80.

6. Wastesson JW, Morin L, Tan ECK, Johnell K. An update on the clinical consequences of polypharmacy in older adults: a narrative review. Expert Opin Drug Saf. 2018;17(12):1185-96.

7. Fried TR, O'Leary J, Towle V, Goldstein MK, Trentalange M, Martin DK. Health Outcomes Associated with Polypharmacy in Community-Dwelling Older Adults: A Systematic Review. J Am Geriatr Soc. 2014;62(12):2261-72.

8. Palmer K, Villani ER, Vetrano DL, Cherubini A, Cruz-Jentoft AJ, Curtin D et al. Association of polypharmacy and hyperpolypharmacy with frailty states: a systematic review and meta-analysis.Eur Geriatr Med . 2019;10(1):9-36.

9. Medication Safety in Polypharmacy. Geneva: World Health Organization; 2019 (WHO/UHC/SDS/2019.11). Licence: CC BY-NC-SA 3.0 IGO

10. Masnoon N, Shakib S, Kalisch-Ellett L, Caughey GE. What is polypharmacy? A systematic review of definitions. BMC Geriatr. 2017;17(1):230.

11. Khanna S, Rolls DA, Boyle J, Xie Y, Jayasena R, Hibbert M, Georgeff M. A risk stratification tool for hospitalisation in Australia using primary care data. Sci Rep. 2019;9(1):5011.

12. Wallace E, Stuart E, Vaughan N, Bennett K, Fahey T, Smith SM. Risk prediction models to predict emergency hospital admission in community-dwelling adults: a systematic review. Med Care. 2014;52(8):751-65.

13. Alhawassi TM, Krass I, Bajorek BV, Pont LG. A systematic review of the prevalence and risk factors for adverse drug reactions in the elderly in the acute care setting. Clin Interv Aging. 2014;9:2079-86.

14. Hartikainen S, Lonnroos E, Louhivuori K. Medication as a risk factor for falls: critical systematic review. J Gerontol A Biol Sci Med Sci 2007;62(10):117281.

15. Zia A, Kamaruzzaman SB, Tan MP. Polypharmacy and falls in older people: Balancing evidence-based medicine against falls risk. Postgrad Med. 2015;127(3):330-7.

16. Reeve E, Shakib S, Hendrix I, Roberts MS, Wiese MD. Review of deprescribing processes and development of an evidence-based, patient-centred deprescribing process. Br J Clin Pharmacol. 2014;78(4):738-47.

17. Pharmaceutical Society of Australia 2019. Medicine Safety: Take Care. Canberra: PSA.

18. Australian Commission on Safety and Quality in Health Care. Continuity of medication management. 2019.

https://www.safetyandquality.gov.au/standards/nsqhs-standards/medication-safety-standard/continuity-medication-management. Accessed 24-03-21

Page $11 / 13$ 
19. Shakib S, Dundon BK, Maddison J, Thomas J, Stanners M, Caughey GE et al. Effect of a Multidisciplinary Outpatient Model of Care on Health Outcomes in Older Patients with Multimorbidity: A Retrospective Case Control Study. PloS one. 2016;11(8):e0161382.

20. Masnoon N, Shakib S, Kalisch-Ellett L, Caughey GE. Tools for Assessment of the Appropriateness of Prescribing and Association with Patient-Related Outcomes: A Systematic Review.Drugs Aging . 2018;35(1):43-60.

21. Masnoon N, Shakib S, Kalisch-Ellett L, Caughey GE. Rationalisation of polypharmacy in practice: a survey of physicians and pharmacists.J Pharm Pract Res . 2019.

22. Australian Medicines Handbook Pty Ltd. Australian Medicines Handbook. 2019. https://amhonline.amh.net.au/auth. 2019. Accessed 24-03-21

23. SA Department of Health and Ageing Safety and Quality Unit. High risk medicines. 2017.

https://www.sahealth.sa.gov.au/wps/wcm/connect/public+content/sa+health+internet/clinical+resources/clinical+topics/medicines+and+drugs/high+risk+m Accessed 06-11-18.

24. American Geriatrics Society. American Geriatrics Society 2015 Updated Beers Criteria for Potentially Inappropriate Medication Use in Older Adults. J Am Geriatr Soc. 2015;63(11):2227-46.

25. Colagiuri S DS, Girgis S, Colagiuri R. Evidence Based Guideline for Blood Glucose Control in Type 2 Diabetes Canberra 2009.

26. Lung Foundation Australia. Australian and New Zealand Guidelines for the management of Chronic Obstructive Pulmonary Disease 2018.

27. Heart Foundation. Australian and New Zealand Guidelines for the management of Chronic Obstructive Pulmonary Disease 2018.

28. Steinman MA. Polypharmacy-Time to Get Beyond Numbers.JAMA Intern Med . 2016;176(4):482-3.

29. Fried TR, Mecca MC. Medication Appropriateness in Vulnerable Older Adults: Healthy Skepticism of Appropriate Polypharmacy.J Am Geriatr Soc . 2019;67(6):1123-7.

30. Sirois C, Tannenbaum C, Gagnon M-E, Milhomme D, Émond V. Monitoring polypharmacy at the population level entails complex decisions: results of a survey of experts in geriatrics and pharmacotherapy. Drugs \& Therapy Perspectives. 2016;32(6):257-64.

31. Parekh N, Ali K, Davies JG, Stevenson JM, Banya W, Nyangoma S et al. Medication-related harm in older adults following hospital discharge: development and validation of a prediction tool.BMJ Qual Saf 2020;29(2):142-153.

32. Sixth Community Pharmacy Agreement. Home Medicines Review. http://6cpa.com.au/medication-management-programs/home-medicines-review/. Accessed 02-10-19.

33. Burt J, Elmore N, Campbell SM, Rodgers S, Avery AJ, Payne RA. Developing a measure of polypharmacy appropriateness in primary care: systematic review and expert consensus study. BMC Med. 2018;16(1):91.

\section{Figures}

Total number of medicines

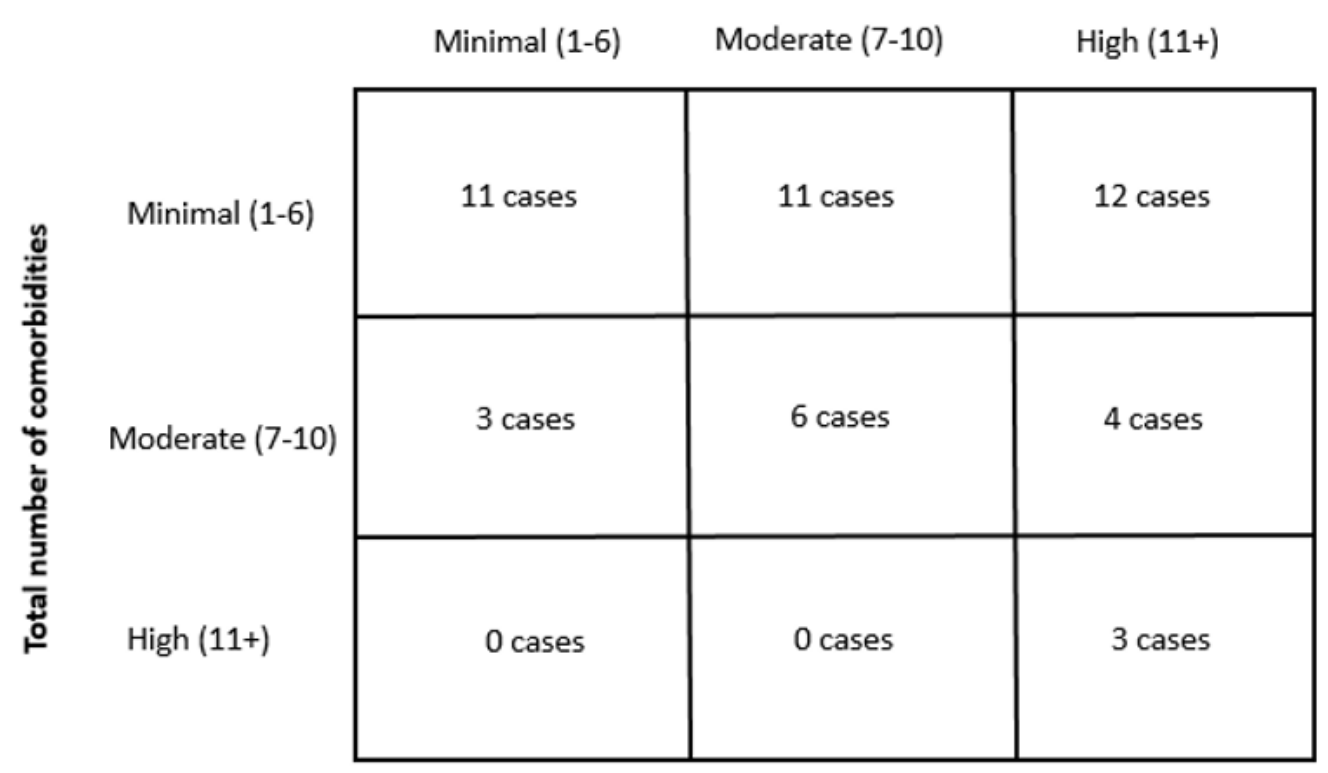

Page $12 / 13$ 
Figure 1

Breakdown of cases according to total number of medications and comorbidities

\section{A Background}

67 year old person living independently in the community

\section{B Comorbidities}

- Back pain

- Atrial fibrillation

- Rheumatoid arthritis

- Depression

- Hypertension

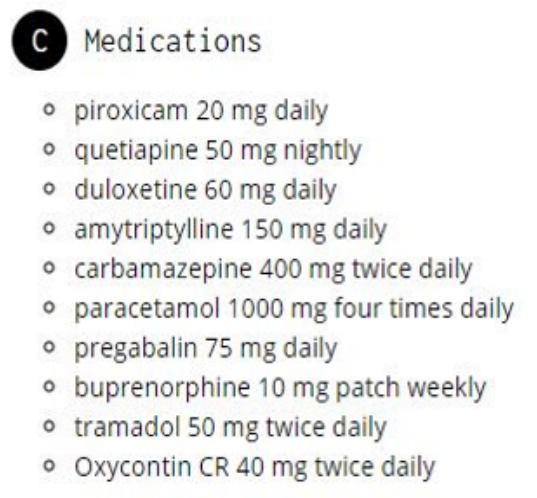

Figure 2

Snapshot of a case on WIPE

\section{Supplementary Files}

This is a list of supplementary files associated with this preprint. Click to download.

- Appendix.docx 